\title{
riccafd
}

Revista Iberoamericana de Ciencias de la Actividad Física y el Deporte

\section{INFLUENCIA DEL PESO Y EL NIVEL DE ACTIVIDAD FÍSICA EN LA CALIDAD DE VIDA DE ESTUDIANTES UNIVERSITARIOS}

\author{
WEIGHT AND LEVELS OF PHYSICAL ACTIVITY INFLUENCES ON \\ QUALITY OF LIFE OF UNIVERSITY STUDENTS
}

Gallego Sánchez-Noriega, J.

Licenciado en Ciencias de la Actividad Física y Deporte. Universidad de Sevilla Correo electrónico: jgallego17@hotmail.com

Código UNESCO: 2402.05

Clasificación Consejo de Europa: 9

Recibido el 30 de marzo de 2014

Aceptado el 12 de abril de 2015

\section{RESUMEN}

Este estudio persigue dilucidar si hay relación existente entre la cantidad de actividad física realizada, la composición corporal y la Calidad de Vida Relacionada con la Salud (CVRS) en estudiantes de primer curso de Grado en Educación Infantil $(n=88)$. Los sujetos realizaron el cuestionario SF-36 sobre CVRS y el cuestionario Internacional de Actividad Física (IPAQ). Los sujetos fueron divididos en tres grupos de acuerdo a su Índice de Masa Corporal (IMC). Los resultados obtenidos muestran que no existe relación existente entre la cantidad de actividad física, la composición corporal y la CVRS en esta muestra.

Palabras clave: Sobrepeso, Obesidad, Calidad de vida, IMC, Composición corporal.

\section{ABSTRACT}

This study aims to elucidate whether the relationship between the amount of physical activity, body composition and Quality of Life Health Related (HRQOL) in freshmen Degree in Early Childhood Education $(n=88)$. Subjects performed the SF-36 questionnaire on HRQOL and the International Physical Activity Questionnaire (IPAQ). The subjects were divided into three groups according to their Body Mass Index (BMI). The results show that there is no relationship between the amount of physical activity, body composition and HRQOL in this sample.

Key words: Overweight, Obesity, HRQoL, BMI, Body composition. 


\section{INTRODUCCIÓN}

En las últimas décadas sobrepeso y obesidad están convirtiéndose en una de las afecciones con más prevalencia en la sociedad española, abarcando a población de todas las edades, sexos y situación socioeconómica'. Extensa bibliografía demuestra que sobrepeso y obesidad son importantes factores de riesgo para otras enfermedades crónicas, tales como hipertensión, enfermedades cardiovasculares o la diabetes mellitus tipo 2, entre otras ${ }^{2}$. Además su impacto en la calidad de vida, mortalidad prematura y el gasto sanitario producidos, han convertido realmente al sobrepeso y la obesidad en problemas de salud pública. Debido a esta situación el grupo de trabajo internacional en obesidad (IOTF) y la Organización Mundial de la Salud (OMS) definieron a la obesidad como la epidemia del s. XXI, por su alta prevalencia, evolución ascendente, alto impacto sobre las enfermedades crónicas y aumento del gasto sanitario.

La SEEDO de acuerdo con la Organización Mundial de la Salud, considera que un sujeto tiene sobrepeso, cuando este tiene un IMC igual o superior a 25 , así como considera obesidad a un IMC igual o superior a 30 . La ciencia también demuestra que sobrepeso/obesidad afectan negativamente a la calidad de vida relacionada con la salud, tanto en población adulta como infantil $^{3-7}$. La calidad de vida relacionada con la salud (CVRS) se puede definir como el estado de salud percibido por los pacientes. Este concepto ha ido adquiriendo peso en la literatura científica, debido a que son las propias percepciones de los sujetos los que definen los resultados. Para ello suelen utilizarse cuestionarios establecidos para cada tipo de enfermedad y que evalúan tanto el estado físico como emocional.

De la mano del aumento desmesurado de la obesidad y su prevalencia, nos encontramos otro factor preocupante como el escaso nivel de actividad física realizado por los jóvenes. En la sociedad española, los niveles de actividad física realizados son bajos tanto en la población infantil, donde entre el 50 y el $80 \%$ de los niños se califica como sedentarios ${ }^{8}$, como en población adulta donde el $57 \%$ de la sociedad española afirma no realizar actividad físicodeportiva ${ }^{9}$ Es de sobra aceptado que buenos niveles de actividad física correlacionan positivamente con la percepción de una calidad de vida mayor y negativamente con un IMC no saludable, tanto en población adulta como en población infantil ${ }^{10-11}$. Sin embargo, no encontramos en la bibliografía muchos trabajos que estudien específicamente los niveles de actividad física de la población universitaria española y los relacionen con la calidad de vida percibida de los mismos. Diversos autores ${ }^{12-14}$ recogieron dentro sus estudios sobre hábitos alimenticios, si los participantes realizaban actividad física o no, con resultados dispares.

Diferentes autores piensan que los futuros profesionales de salud deben incorporar la práctica de ejercicio físico de forma moderada en su estilo de vida, debido a que no podrán hacer promoción de algo que no practican ${ }^{13}$. De la misma manera los futuros profesionales de la educación, especialmente de

2 INFLUENCIA DEL PESO Y EL NIVEL DE ACTIVIDAD FÍSICA EN LA CALIDAD DE VIDA DE ESTUDIANTES UNIVERSITARIOS 
la Educación Infantil y Educación Primaria, deberían promover activamente la práctica de actividad física y tener una buena actitud ante ello, ya que son los principales agentes secundarios de la socialización del alumnado.

Por ello el objetivo fundamental de este estudio ha sido determinar la relación existente entre cantidad de actividad física, Índice de Masa Corporal (IMC) y su influencia en la Calidad de vida relacionada con la salud, en estudiantes de Primer curso de Grado en Educación Infantil de la Facultad de Educación de la Universidad de Sevilla. En relación con el objetivo anterior se ha estudiado como la práctica de AF afecta a diferentes componentes de la salud física y la salud mental.

\section{MATERIAL Y MÉTODOS}

\section{Participantes}

La muestra escogida para esta investigación han sido 88 alumnos de primer curso del Grado en Educación Infantil de la Facultad de Ciencias de la Educación de la Universidad de Sevilla, todas ellas mujeres. La edad de la muestra está comprendida entre los 18 y 25 años. Los únicos criterios de inclusión que se han considerado en este estudio es pertenecer al primer curso de Grado en Infantil de la Universidad de Sevilla y tener menos de 28 años. Todos los sujetos cumplían estos requisitos y pudieron completar el estudio.

\section{Medidas}

Las medidas socio-demográficas (edad y sexo) y las antropométricas (talla, peso e IMC) fueron recogidas en una hoja-cuestionario diseñada para tal fin. El Índice de Masa Corporal (IMC) es considerado como la relación entre el peso en kilogramos y la talla en metros al cuadrado, y es utilizado para determinar el sobrepeso en población adulta. De acuerdo a la determinación del IMC y a los criterios de la SEEDO, se ha dividido la muestra en 3 grupos diferentes, peso insuficiente, normopeso, y sobrepeso, <18,5; 18,5-24,9; 2529,9; respectivamente. El nivel de actividad física y sedentarismo fueron estudiados a través del Cuestionario Internacional de Actividad Física (IPAQ). EI IPAQ estima la actividad física realizada, preguntando el número de días y las horas y minutos empleados en una semana en actividad física, dividiendo la misma entre actividad vigorosa, moderada y caminar. El sedentarismo se estima a través de la pregunta del número de horas sentadas que una persona puede pasar al día. Para evaluar la Calidad de Vida Relacionada con la Salud (CVRS) se utilizó el cuestionario SF-36 versión española. Este cuestionario está compuesto por 36 ítems divididos en 8 dimensiones. De las 8 dimensiones, 4 se corresponden al componente físico y las otras 4 al componente mental. Las puntuaciones van de 0 a 100, siendo 0 signo de muy mala calidad de vida y 100 de muy buena. 
Tabla 1. Características de los participantes en el estudio $(n=88)$

\begin{tabular}{|c|c|}
\hline Variables & Media(DE) \\
\hline Peso (kg) & $58,78(8,91)$ \\
\hline Altura (m) & $1,62(0,05)$ \\
\hline Edad(años) & $21,46(2,99)$ \\
\hline IMC (kg/m $\left.{ }^{2}\right)$ & $22,22(3,18)$ \\
\hline \multicolumn{2}{|c|}{ Variables presentadas como Media (DE) y IMC: Índice de masa corporal }
\end{tabular}

\section{Procedimiento}

Una vez elegida la muestra para este estudio, los investigadores se reunieron con los sujetos, que contestaron cuestionarios IPAQ, SF-36 y una serie de preguntas socio-demográficas, que les fue entregado en una hoja conjunta. Los investigadores explicaron el procedimiento a seguir y resolvieron las dudas que se plantearon. Con los resultados de estatura y peso, posteriormente se aplicó la fórmula peso $(\mathrm{kg}) /$ altura^2 $(\mathrm{m})$ para lograr el IMC y dividir los resultados en 3 grupos distintos.

\section{Análisis estadístico}

Para realizar el análisis estadístico de esta investigación se usó el programa SPSS 19.0. Se estableció un nivel de significación de ${ }_{p}<0,5$ en todos los análisis realizados. Se realizó un análisis descriptivo de todas las variables incluidas en el estudio. La estadística descriptiva fue presentada como media y DE. Para observar las diferencias entre grupos se realizó un test de varianza ANOVA

\section{RESULTADOS}

En la tabla 1, se observan los valores de las medidas obtenidas de la muestra. Un total de 88 estudiantes de primer curso de Grado en Educación Infantil, todas ellas mujeres, fueron analizadas en este estudio.

En la tabla 2, puede observarse el nivel de correlación existente entre la CVRS, la cantidad de actividad física, el sedentarismo y la composición corporal medida con IMC. Solo se observa correlación significativa en las columnas que se corresponde con los minutos totales de caminar y el sedentarismo. Más concretamente se observa como la dimensión de función social está correlacionado negativamente con los minutos totales de caminata a la semana. Por su parte la dimensión salud mental también está correlacionada negativamente con los minutos de caminata, así como el componente global de salud mental. Por contrapunto se observa correlación positiva entre el tiempo de sedentarismo y las dimensiones función social, salud mental y el componente de estado mental global. El IMC no está relacionado ni con el componente físico ni con el mental. 
Tabla 2 Correlación entre CVRS y el nivel de actividad física, sedentarismo e IMC ( $\mathrm{n=88}$ )

\begin{tabular}{|c|c|c|c|c|c|}
\hline \multicolumn{6}{|c|}{ ACTIVIDAD FÍSICA Y SEDENTARISMO: IPAQ } \\
\hline CVRS & $\begin{array}{c}\text { Tiempo total de } \\
\text { actividad } \\
\text { Fisica/Semana } \\
\text { (minutos/semanas) }\end{array}$ & $\begin{array}{c}\text { Actividad fisica } \\
\text { total /semana } \\
\text { (MET's/minuto/semana) }\end{array}$ & $\begin{array}{l}\text { Total Min } \\
\text { Caminar }\end{array}$ & $\begin{array}{c}\text { Tiempo } \\
\text { total/sentado } \\
\text { semana } \\
\text { (minutos/semana) }\end{array}$ & IMC \\
\hline $\begin{array}{l}\text { Función } \\
\text { Física }\end{array}$ & 071 & 105 & 077 &,- 061 & $\overline{-}$ \\
\hline Rol Físico &,- 020 &,- 045 & ,005 & , 079 & $\overline{163}$ \\
\hline $\begin{array}{l}\text { Dolor } \\
\text { corporal }\end{array}$ &,- 057 &,- 011 &,- 163 &,- 052 &, 074 \\
\hline $\begin{array}{l}\text { Salud } \\
\text { General }\end{array}$ &,- 100 &,- 049 &,- 075 & ,061 & , 164 \\
\hline Vitalidad & 054 & 026 & ,021 &,- 010 & , 114 \\
\hline $\begin{array}{l}\text { Función } \\
\text { social }\end{array}$ &,- 141 &,- 132 &,$- 211^{*}$ & $244^{\circ}$ & ,045 \\
\hline $\begin{array}{l}\text { Rol } \\
\text { emocional }\end{array}$ &,- 071 &,- 018 &,$- 234^{*}$ & .182 &, 027 \\
\hline $\begin{array}{l}\text { Salud } \\
\text { mental }\end{array}$ &,- 175 &,- 153 &,$- 248^{\circ}$ & ,243. & ,080 \\
\hline $\begin{array}{l}\text { Salud } \\
\text { Fisica } \\
\text { Global } \\
\text { Salud }\end{array}$ & 025 & 026 & ,096 &,- 120 & $\overline{-}, 102$ \\
\hline $\begin{array}{l}\text { Mental } \\
\text { Global }\end{array}$ &,- 129 &,- 096 &,$- 276 "$ &, $261^{\circ}$ & $\overline{0}, 0$ \\
\hline
\end{tabular}

Coeficientes de correlación de Pearson; CVRS: Calidad de Vida relacionada con la Salud SF36: Cuestionario especifico de Calidad de Vida relacionada con la Salud en población general; IPAQ: Cuestionario Internacional de Actividad Física; IMC: Índice de Masa Corporal; ${ }^{* *}$. La correlación es significativa en nivel $0,01 .{ }^{*}$. La correlación es significativa en el nivel 0,05.

Como puede observarse en la tabla 3 , no existen diferencias significativas entre los resultados de los grupos diferenciados por IMC.

Tabla 3. Diferencias de CVRS entre los grupos de IMC, comparando los resultados del cuestionario SF-36

\begin{tabular}{|l|c|c|c|}
\hline CVRS: SF-36 & IMC <18,5 (n=9) & $\begin{array}{c}\text { IMC 18,5-24,9 } \\
(\mathbf{n = 6 6 )}\end{array}$ & $\begin{array}{c}\text { IMC 25-30 } \\
(\mathbf{n = 1 1 )}\end{array}$ \\
\hline SALUD FÍSICA GLOBAL & $50,52(5,27)$ & $51,93(5,42)$ & $48,95(6,72)$ \\
\hline Función física & $93,33(6,61)$ & $95,00(6,44)$ & $92,89(8,22)$ \\
\hline Rol físico & $91,67(25,00)$ & $90,83(19,51)$ & $76,32(30,59)$ \\
\hline Dolor corporal & $57,78(10,63)$ & $70,70(17,36)$ & $68,68(22,63)$ \\
\hline Salud general & $85,18(33,79)$ & $65,48(15,62)$ & $58,05(15,27)$ \\
\hline SALUD MENTAL GLOBAL & $45,75(11,53)$ & $46,38(8,51)$ & $44,89(10,44)$ \\
\hline Vitalidad & $68,00(17,32)$ & $60,67(13,69)$ & 53,194 \\
\hline Función social & $63,89(22,96)$ & $88,13(14,45)$ & $78,29(24,24)$ \\
\hline Rol emocional & $81,94(23,48)$ & $77,78(33,99)$ & $0,15,33)$ \\
\hline Salud Mental & $63,4(14,31)$ & $73,00(11,93)$ & 0,101 \\
\hline
\end{tabular}


*Valores expresados como Media (DE); CVRS: Calidad de vida relacionado con la salud SF-36: Cuestionario específico de Calidad de vida relacionado con la salud en población general; IPAQ: Cuestionario Internacional de Actividad Física; IMC: Índice de Masa Corporal. * $\mathrm{p}$ valor significativo en el nivel .05. ${ }^{* *} \mathrm{p}$ valor significativo en el nivel .01

\section{DISCUSIÓN}

Pese a que existe una enorme cantidad de estudios que afirman que la CVRS se ve influenciada negativamente por el peso inadecuado y la escasa cantidad de actividad física en población general ${ }^{15,3}$ en este estudio y con esta población en concreto no se han encontrado resultados realmente significativos, posiblemente debido a que el número de sujetos en los diferentes grupos no fue proporcional y la muestra total cuantitativamente fue escasa. La predominancia del grupo de normopeso era evidente, por lo que los resultados de su grupo y en consonancia con lo que muestra la literatura, no iba a reportar ningún dato de interés. Para que este estudio hubiese tenido una mayor posibilidad de encontrar datos estadísticamente significativos se debería haber propuesto que uno de los criterios de inclusión hubiese sido estar en situación de sobrepeso (IMC>24,9).

Aunque no son datos estadísticamente significativos y no se pueden sacar conclusiones sobre estos datos sí que se observa descenso de los componentes estado físico general y estado mental general, en el grupo con sobrepeso $[48,95(6,72)$ y $44,89(10,44)]$ respecto al grupo de normopeso $[51,93(5,42)$ y $46,38(8,51)]$. Uno de los datos más llamativos que encontramos en este estudio, es que habiendo escogido una muestra de forma aleatoria de una población específica, se han encontrado que el 10,2\% de la muestra estaba dentro del grupo de peso insuficiente, que también puede hacer surgir problemas en la calidad de vida ${ }^{16-17}$.Sin embargo estos datos no son preocupantes, ya que no eran datos extremos y no es raro encontrarse con casos de mujeres con un IMC menor de 18.5 sin que exista ninguna anomalía en su salud, por lo que por eso mismo tampoco llegaba a influir en los resultados de la CVRS. De nuevo, observamos que aunque los datos no son significativos, este grupo obtuvo la peor puntuación de los 3 en la dimensión salud mental $[63,4(14,31)]$ en comparación con el resultado del grupo normopeso $[73,00(11,93)]$

Analizándose los datos obtenidos con la investigación se puede observar como los resultados obtenidos en los cuestionarios son moderados. El cuestionario SF-36, en su análisis se compone de 8 dimensiones y dos componentes finales, estado físico y estado mental. Se establecen unas puntuaciones de 0 a 100 , siendo 0 muy mal estado de salud y 100 , perfecto. Como podemos observar en la tabla 3 de la varianza ANOVA, los datos principales que son los de estado de salud física general y el estado de salud mental, están en torno a los 50 puntos o ni siquiera llegan a eso, tanto el grupo de peso inferior al recomendado, al de normopeso y al de sobrepeso. Este es un indicativo de que no se sienten realmente bien tanto físicamente como mentalmente. Puede ser debido a multitud de factores, pero si se sigue observando la tabla, se pueden observar otros datos interesantes. Las 
dimensiones que corresponden al apartado físico parecen tener buena puntuación, sin embargo, la dimensión dolor corporal, tiene peores resultados que los otros. Se puede intuir que los participantes en la investigación, tenían o tienen dolores frecuentes, por motivos que se desconocen. En cuanto al apartado mental o psicológico, la dimensión con peor puntuación es la de vitalidad. La muestra de este estudio estaba compuesta por estudiantes de primer curso y el comienzo de una etapa nueva de su vida, como es la universidad puede llegar a ser traumático. Además habría que tomar en cuenta el contexto y la temporalidad en la que se realizó la investigación: en el primer período de exámenes del primer cuatrimestre del curso.

Precisamente y lo que sí está claro, es que el grupo que menor vitalidad parece tener es el grupo con sobrepeso. Los motivos no correlacionan estadísticamente con los de actividad física o con su composición corporal, pero se puede considerar como dato de importancia y es relevante, ya que la única diferencia aparente con el resto de compañeros, son la composición corporal, es decir el sobrepeso que sufren.

Las únicas correlaciones significativas que se han encontrado también son llamativas. Se ha encontrado correlación negativa entre los minutos de caminata a la semana y la función social y el componente mental y correlación positiva entre el sedentarismo y la función social. Según los datos se puede interpretar que los minutos caminando a la semana se realizan en solitario, por deporte o por desplazamiento. Por otro lado también podemos interpretar que los minutos sentados o de sedentarismo se hacen en compañía, de ahí la correlación positiva entre estas dos variables.

\section{CONCLUSIONES}

Según los resultados encontrados en este estudio, la cantidad de actividad física y la composición corporal no afectan al estado físico ni mental de los estudiantes de primer curso en Grado en Educación Infantil de la Facultad de Ciencias de la Educación de la Universidad de Sevilla.

El estudio puede servir como punto de partida para nuevas investigaciones en población universitaria. Como se ha comentado, tantos profesionales de la salud como de la Educación, deben servir de ejemplos para sus "clientes". Por ello mismo es necesario estudiar aún más si existe relación entre relación entre la CVRS, la cantidad de actividad física y la composición corporal y si esto puede repercutir en un futuro en la educación del alumnado.

\section{REFERENCIAS BIBLIOGRÁFICAS}

1. Aranceta J, Perez Rodrigo C, Serra Majem, L. (2003). Prevalencia de la obesidad en España: resultados del estudio SEEDO 2000. Med Clin (Barc).120: 608-612.

2. Must A, Spadano J, Coakley EH, Field AE, Colditz G, Dietz WH. (1999). The disease burden associated with overweight and obesity. JAMA. 282(16): 1523-9.

3. Dey, M., Gmel, G., Mohler-Kuo, M., (2013). Body mass index and health-related quality of life among young Swiss men. BMC Public Health. 13:1028

4. Yancy WS Jr, Olsen MK, Westman EC, Bosworth HB, Edelman D. (2002). Relationship 
between obesity and health-related quality of life in men. Obes Res. 10(10):1057 64.

5. Huang IC, Frangakis C, Wu AW. (2006). The relationship of excess body weight and healthrelated quality of life: evidence from a population study in Taiwan. Int J Obes (Lond). 30(8):1250-9.

6. Schwimmer JB, Burwinkle TM, Varni JW. (2003). Health-related quality of life of severely obese children and adolescents. JAMA. 289(14):1813-9

7. Cárdenas, VM; Dávila, S.; Gallegos, E.; Salazar, BC.; Rizo, MM. (2009). Obesidad y Calidad de Vida Relacionada con la Salud en Adolescentes Escolares. Desarrollo Cientif. Enferm. 17 (7)

8. Encuesta Nacional de Salud 2006. Madrid: Ministerio de Sanidad y Consumo. Secretaría General Técnica. Centro de publicaciones. Disponible en www.msps.es y en www.ine.es/inebase

9. García Ferrando, M, Llopis Goig, R., Encuesta sobre los hábitos deportivos en España 2010. Disponible en: www.csd.gob.es/csd/estaticos/dep-soc/encuesta-habitosdeportivos2010.pdf

10. Muros-Molina, J., Som-Castillo, A., López, H., Zabala, M. (2009). Asociaciones entre el IMC, la realización de actividad física y la calidad de vida en adolescentes. Cultura, Ciencia y Deporte. 4 (12), p.p. 159-165

11. Del Hoyo, M, Sañudo, B., (2007). Composición corporal y actividad física como parámetros de salud en niños de una población rural de Sevilla. Revista Internacional de Ciencias del Deporte. 3 (6) p.p.: 52-62

12. Ledo-Varela, M.T., de Luis Román, D.A., González-Sagrado, M., Izaola Jauregui, O., Conde Vicente, R. y Aller de la Fuente, R. (2011). Nutr Hosp. 26(4):814-818

13. Bayona Marzo I, Navas-Cámara FJ, Fernández de Santiago FJ, Mingo-Gómez T, de la Fuente Sanz MM, Cacho del Amo A. (2007). Hábitos dietéticos en estudiantes de fisioterapia. Nutr Hosp. (22 (5): 573-7

14. Martínez Roldán C, Veiga Herreros P, López de Andrés A, Cobo Sanz JM, Carbajal Azcona A. (2005). Evaluación del estado nutricional de un grupo de estudiantes universitarios mediante parámetros dietéticos y de composición corporal. Nutr Hosp 20: $197-203$.

15. Latorre, P.A.; Salas, J., Soto, V.M., (2012). Composición corporal relacionada con la salud en atletas veteranos. Nutr Hosp. 27(4) p.p.: 1236-1243

16. van Grieken, A., Renders, C.M., Wijtzes, A.I., Hirasing, R.A., Raat, H. (2013). Overweight, obesity and underweight is associated with adverse psychosocial and physical health outcomes among 7-year-old children: the 'Be active, eat right' study. PLoS ONE. 8(6): e67383. doi:10.1371/journal.pone.0067383

17. Adair, CE., Marcoux, GC., Bischoff, TF., Cram, BS., Ewashen, CJ., Pinzon, J., Gusella, JL., Geller, J., Scattolon, Y., Fergusson, P., Styles, L., Brown, KE. (2010). Health and Quality of Life Outcomes. 8:83

Referencias totales citadas: 17 .

Referencias citadas correspondientes a la Rev Ib CC Act Fis Dep: 0. 